\title{
Prendre soin du sujet de l'éducation
}

\section{(2) OpenEdition}

Journals

Édition électronique

URL : https://journals.openedition.org/leportique/912

DOI : 10.4000/leportique.912

ISSN : $1777-5280$

\section{Éditeur}

Association "Les Amis du Portique"

\section{Référence électronique}

"Prendre soin du sujet de l'éducation », Le Portique [En ligne], e-Portique, mis en ligne le 08 janvier 2007, consulté le 10 septembre 2022. URL : http://journals.openedition.org/leportique/912 ; DOI : https://doi.org/10.4000/leportique.912

Ce document a été généré automatiquement le 10 septembre 2022

Tous droits réservés 


\section{Prendre soin du sujet de l'éducation}

«Vérifions la double hypothèse suivante : le soin relève de l'art, entendu au sens de la technê, grecque, et l'art repose, quant à lui, sur un jugement pratique, particulier et singulier, dont rend au mieux compte l'expérience insolite du tact. »

(JP Resweber, "Le soin : affaire de technê et de tact", Les Gestes de soin).

1 Entre préoccupation et occupation, culture et traitement, attention et responsabilité, le soin est au coeur de l'entreprise éducative.

2 Idée directrice de l'anthropologie, thérapeutique du corps et de l'âme, le soin, rapporté aux trois ordres de la Cura comme exercice inquiet du souci du monde, d'autrui et de soi, de la Cure comme vigilance, catharsis et thérapeutique, du Care comme geste de sollicitude ${ }^{1,}$ est le modèle des pratiques de veille, d'intervention ou de réflexion, et, concernant l'éducation, d'enseignement et de pédagogie. Le geste de soin ${ }^{2}$ est l'art, au sens de la technè grecque, d'une mise en oeuvre du tact..

4 L'éthique du soin ne s'accommode pas d'échappatoire : pour reprendre un mot de Savater, dans la tradition stoïcienne : "si ce qui nous offusque ou nous préoccupe peut être changé, nous devons mettre la main à la pâte ; et si c'est impossible, alors il est oiseux de le déplorer"3. C'est pourquoi il nous faut prendre soin, avec attention, prudence et bienveillance. Mais si "soigner"c'est remédier aux dysfonctionnements, ou prévenir les dérives, c'est aussi engager l'action et convoquer exigence de réflexivité et philosophie pour vivre. Le soin est alors attention à la santé conçue comme "bonheur humain" (soin/sain/saint). Il s'agit d'une cause commune : mais si tous s'accordent sur sa nécessité, encore faut-il savoir comment une "philosophie du soin" peut s'articuler à l'authenticité de l'action.

5 Pour aller plus loin, il nous faut actualiser la nécessité du soin - qui touche ce à quoi profondément nous aspirons - ${ }^{4}$ et souligner son caractère consubstantiel à l'éducation comprise comme moment primordial du processus d'hominisation. En cela, le soin se rapporte à une stratégie générale de nous-mêmes sur nous-mêmes : veiller à ce que nous sommes, et à ce que nous devenons. Tout n'est jamais gagné, et nous savons les risques, les atteintes, les négligences et les reculades qui nous dispensent trop souvent d'une référence à l'éthique, attentive et constante. 
Ce dossier réunit, en une rencontre interdisciplinaire, des spécialistes de différents domaines d'intervention. Les contributions, portant sur diverses dimensions théorétiques et praxéologiques, ont été regroupées en deux tomes.

Cette première livraison présente deux ensembles : un groupe de textes en liminaire situe les principes philosophiques (historiques, théorétiques) d'une question que la seconde partie examine selon différentes références en éducation de base : ouverture à l'imaginaire ou rapport au corps, actes primordiaux ou situation scolaire, modèles de prise en compte de l'altérité.

8 Sur cette même articulation le second tome s'attache à contextualiser des pratiques sous divers angles (anthropologique, sociologique, pédagogique), en habilitant le sujet du soin dans sa raison d'être.

Que les auteurs qui ont accepté de participer à cette réflexion commune soient ici remerciés.

\section{NOTES}

1. Ainsi du Prendre soin (Hesbeen), du Care (Tronto), du Souci - "du monde" (Arendt), - ou "de soi " (Foucault), du Sens illatif (Newman).

2. Ce dossier fait écho au travail du séminaire Anthropologie du soin qui s'est tenu à l'université de Metz en 2002-2003 dans le cadre du laboratoire ERASE, et dont a témoigné la publication Les Gestes de soin, Les Cahiers du Portique, 2003 (s/d J.-P. Resweber). http:/ leportique.revues.org/document905.html

3. De l'éducation, Payot et Rivages, 1998

4. "Le soin de l'âme - la philosophie le répète de Socrate à Rousseau, Kant ou Patocka - est le seul risque pour l'homme qui vaille la peine d'être couru". J.-F. Mattéi, La philosophie de l'éducation ou le paradoxe pédagogique (1994). 